\section{Aspectos de la fecundidad de mujeres indígenas Suruí, Rondônia, Brasil: una aproximación}

\section{Aspects of the fertility of indigenous Surui women in Rondônia, Brazil: an approximation}

María Mercedes Arias Valencia 1

Ricardo Ventura Santos 2

Carlos E.A. Coimbra Jr. 3

Maurício V.G. Oliveira 4

Ana Lúcia Escobar 5

1 Sistema Universitario de Investigación. Universidad de Antioquia. Antioquia, Colombia.

2-4 Escola Nacional de Saúde Pública. Fundação Oswaldo Cruz. Rua Leopoldo Bulhões, 1480. Manguinhos. Rio de Janeiro, RJ, Brasil. CEP: 21041-210. E-mail: coimbra@ensp.fiocruz.br

5 Centro de Estudos em Saúde do Indio de Rondônia/ CESIR. Universidade Federal de Rondônia. Porto Velho, RO, Brasil.

\begin{abstract}
Objectives: to analyze female fertility among Surui Indian women in the Brazilian Amazon.

Methods: a total of 88 reproductive and birth histories were collected by means of household interviews carried out in two Surui villages in 2004.

Results: the average age of menarche was 12 years-old; the average ages of first marriage and of the delivering the first child were, respectively, 13.8 and 15.7 years-old. For most women, delivery took place in their own village. The average time between pregnancies was 22.8 months and the average breast feeding duration was 18.4 months. Very few Surui women use contraceptives and most have attended school for only a couple of years. On average, the total fertility rate was of 6.3 children per woman.

Conclusions: in comparison with other populations, Surui's demography shows a moderately high fertility rate. The authors argue that the findings of this study can be of use to better plan health services that are more culturally compatible with the reality of Surui women.
\end{abstract}

Key words Fertility, Indians, South American, Brazil

\section{Resumen}

Objetivos: caracterizar aspectos de la fecundidad de mujeres indígenas Suruí de la Amazonía brasilera.

Métodos: se levantaron y sistematizaron 88 historias reproductivas y de nacimientos, obtenidas a través de visitas domiciliarias en dos aldeas, realizadas en 2004.

Resultados:el promedio de edad de la menarquía fue 12 años; las edades medias de entrada a la primera unión y del nacimiento del primer hijo fueron, respectivamente, 13,8 y 15,7 años. El parto ocurrió en la aldea en su mayoría. Las mujeres Suruí presentan un intervalo intergenésico promedio próximo a los 22,8 meses. El promedio de amamantación fue 18,4 meses. El uso de contraceptivos es mínimo y el número de años de estudio de las mujeres es bajo. La tasa de fecundidad total fue de 6,3 hijos.

Conclusión: si se compara con resultados de otros estudios en pueblos indígenas, la demografía Surui señala niveles de fecundidad entre moderados y elevados. Los autores argumentan que los hallazgos son relevantes para la prestación de servicios de salud culturalmente compatibles con la realidad Suruí.

Palabras-clave Fecundidad, Indio Sudamericanos, Brasil 


\section{Introducción}

La antropología demográfica de las sociedades indígenas se ha consolidado en las últimas décadas como campo científico bien delimitado desde el punto de vista teórico-metodológico y ha proporcionado importante contribución al conocimiento sobre la dinámica poblacional de sociedades de pequeña escala.1,2 Además, tales investigaciones han generado datos referentes a la evaluación de las condiciones de salud de dichas poblaciones. Un buen ejemplo de la expansión y consolidación de las investigaciones en antropología demográfica puede verse en los análisis sobre pueblos indígenas en Brasil, y de la cuenca Amazónica en particular, realizadas en las dos últimas décadas. 3-6

Respecto de la dinámica reproductiva, los sistemas socioculturales de los pueblos indígenas de la Amazonía presentan elaborados ordenamientos relativos al inicio de la actividad sexual, edad para el matrimonio, nacimiento del primer hijo, intervalos intergenésicos, finalización del período reproductivo, y así sucesivamente. A pesar de la gran sociodiversidad que presentan los pueblos indígenas en Brasil, con cerca de 220 etnias, lo que en gran medida impide generalizaciones, podemos decir que, en general, las condiciones relacionadas con la salud reproductiva favorecen un cuadro de elevada fecundidad, en el cual, no son escasas tasas totales de fecundidad (TFT) del orden de 7-8 hijos. El patrón de alta fecundidad se relaciona con intervalos intergenésicos cortos, combinados con la iniciación de la fase reproductiva al inicio de la segunda década de la vida, alrededor de los 13-14 años, que se extiende, no raramente, hasta los 40-45 años.3,4,6-9 Desde el punto de vista epidemiológico, elevadas TFT, maternidad temprana en la adolescencia y vida reproductiva de larga duración constituyen una combinación que puede poner a la mujer indígena en general en una posición de vulnerabilidad social y de salud.

Hasta hace pocos años, las estadísticas vitales no estaban disponibles para la amplia mayoría de los pueblos indígenas en Brasil y los aspectos más básicos de su dinámica demográfica eran prácticamente desconocidos.6,10,11 En ese sentido, Coimbra Jr. e Santos 10 llamaron la atención hacia las implicaciones de lo que denominaron una "dañina invisibilidad demográfica y epidemiológica". Dicho panorama ha cambiado en los últimos años, cuando las investigaciones sobre la demografía de los pueblos indígenas en Brasil han tenido una expansión sustancial. Además de investigaciones en comunidades específicas, ${ }^{6}$ los dos últimos censos nacionales realizados en 1991 y 2000 han aportado bases de datos fundamentales para dichas investigaciones. Se resalta que la categoría "indígena" fue incluida en el censo brasileño solamente en 1991, y se capta según el criterio de autodeclaración, con base en la categoría color/raza. ${ }^{5}$

Con miras a contribuir en la literatura sobre demografía y salud reproductiva de pueblos indígenas en Brasil, el presente artículo muestra los resultados de un estudio realizado entre el pueblo Suruí, localizado en Rondônia, en la Amazonía brasilera, enfocando aspectos ligados con la dinámica de la fecundidad y sus determinantes.

\section{Métodos}

Los Suruí, también conocidos por Pahíter, hablan un lenguaje clasificado dentro del tronco lingüístico Tupí, familia Mondé, y están localizados en el sudeste de Rondônia y noroeste de Mato Grosso, en la Terra Indígena Sete de Setembro (aproximadamente, $61^{\circ}$ longitud oeste y $11^{\circ}$ latitud sur). El contacto permanente de los Suruí con la sociedad brasilera ocurrió en 1969, cuando frentes económicos y demográficos nacionales se expandieron en aquella región de la Amazonía brasilera. El contacto vino asociado con alta mortalidad debido a epidemias de sarampión, gripa y tuberculosis, que diezmaron más de la mitad de la población original. 12

Por ocasión de la investigación de campo en 2004, la población Suruí era de aproximadamente 990 habitantes, distribuida a lo largo de 12 aldeas, en su mayoría, situadas al sur de la reserva indígena. Un pequeño número de familias viven en ciudades próximas a la reserva, principalmente Riozinho, pero mantienen contacto con sus familiares en las aldeas. Hay pocos estudios etnográficos realizados sobre los Suruí. ${ }^{13,14}$ Prevalecen en la literatura estudios sobre salud y nutrición, que llaman la atención para el acelerado proceso de transición nutricional y elevada incidencia de tuberculosis. ${ }^{12,15-17}$

Actualmente los Suruí visitan, con frecuencia, las ciudades acercadas a sus tierras. Hay líneas de transporte (bus) regular entre las aldeas y los centros urbanos próximos. Las actividades económicas incluyen agricultura, artesanía y, en forma preocupante, la venta de madera. Además, un número creciente de jóvenes frecuentan escuelas y varios adultos de sexo masculino ejercen actividades asalariadas, a través de instituciones como la Fundação Nacional do Índio (FUNAI) y la Fundação Nacional de Saúde (FUNASA).

La investigación de campo tomó como punto de partida las listas poblacionales puestas a disposición 
por la coordinación regional de la FUNASA de Rondônia. Dichas listas son bastante detalladas y periódicamente actualizadas por los equipos de salud que actúan en el llamado polo-base Cacoal, ligado al Distrito Sanitário Especial Indígena (DSEI) Vilhena, que presta atención de salud a los Suruí. Se recolectaron los siguientes datos de las listas: localización de las aldeas, edad, sexo, composición de los domicilios, estado marital y escolaridad.

El conjunto principal de datos se obtuvo a través de investigación de campo realizada en las aldeas Linha 14 y Placa, en septiembre de 2004, durante la cual se entrevistaron el mayor número posible de mujeres a partir de 10 años de edad; se levantaron también los nacidos vivos y otros datos demográficos correspondientes al año 2003. En las aldeas Linha 14 y Placa, se visitaron la totalidad de las 30 unidades domiciliares, en las cuales levantamos 88 historias reproductivas.

El instrumento que se aplicó para reconstruir la experiencia reproductiva fue la historia reproductiva, que parte de la historia de nacimientos. En cada domicilio se levantaron las historias de las mujeres que aceptaron la entrevista (ninguna mujer rehusó la entrevista). Se investigó la historia de cada hijo nacido vivo y cada uno de los eventos obstétricos desde la menarquía hasta el día de la entrevista. El instrumento de recolección es convencional para la información demográfica que había sido probado y empleado en estudios anteriores ${ }^{7} \mathrm{y}$, en el estudio Suruí, se trata de una aplicación de técnicas recomendadas internacionalmente.18 La guía contempló preguntas específicas y sistemáticas sobre: a) edad de la primera unión conyugal; b) edad de la madre a inicio de la primera gestación y al nacimiento del primer hijo y de los subsecuentes; c) si el parto fue en la aldea u hospitalario; d) caracterización de las prácticas de amamantación; e) ocurrencia de separaciones (o viudez) y nuevas uniones conyugales; f) experiencia reproductiva cuando ocurrieron nuevas uniones; g) en el caso de muerte de niños, edad de la madre y del niño en el caso y por ocasión del evento; h) número deseado de hijos, preferencia por el sexo de ellos; i) uso de contraceptivos.

Las entrevistas las realizó uno de los autores (Arias), en portugués, en el lugar designado por las mujeres, con el fin de garantizar la máxima privacidad. Es importante mencionar que, en la actualidad, casi todas las mujeres Suruí son bilingües (lengua Suruí y portugués). Cuando fue necesario, se pidió ayuda de agentes indígenas de salud de sexo femenino, que viven y trabajan en las comunidades. Sin embargo, no se descartan posibles errores, resul- tantes de la formulación o el entendimiento incorrecto de las preguntas, dadas las distancias lingüísticas y culturales entre los Suruí y los investigadores.

A partir de los datos recogidos en los censos y las entrevistas sobre historia reproductiva, se derivaron: tasas de fecundidad específicas por edad y tasa de fecundidad total; edad media de entrada en la primera unión, de inicio de la primera gestación y de nacimiento del primer hijo; promedio de intervalos intergenésicos; tiempo promedio de amamentación y determinantes de la fecundidad. El análisis de la fecundidad se realizó a través de la verificación del nivel (quantum), dado por las tasas y por la estructura. Con referencia al comportamiento reproductivo, se destacan las variables intermedias y de calendario (timing).

El calendario reproductivo comprende los promedios de edades de menarquía, de entrada a la unión, de inicio de la primera gestación y edad al nacimiento del primer hijo, intervalos intergenésicos y tiempo de amamantación del niño. Los intervalos intergenésicos que se presentan, se calcularon entre el fin de un evento obstétrico y el comienzo de otro, es decir, los períodos sin gestación; esto para calcular el tiempo de recuperación de la madre y el cuidado más exclusivo del niño que nace, así como para contabilizar los tiempos de las gestaciones que terminaron en aborto. Los promedios de intervalos intergenésicos y de lactancia se calcularon para cada madre; por lo tanto, se presenta la distribución que se observa en los promedios individuales.

Se aplicaron las definiciones convencionales de la demografía. El levantamiento de los numeradores, el cálculo de los denominadores y los análisis de los datos siguieron los procedimientos regularmente empleados en estudios de demografía antropológica. 7,19

Las bases de datos se digitaron y depuraron con ayuda del programa Excel y los procedimientos automáticos para los cálculos y el análisis estadístico de resultados se realizaron con ayuda del programa SPSS (Statistical Package for the Social Sciences, v. 14.0). Se utilizaron también planillas de análisis demográfico desarrolladas por la Escola Nacional de Ciências Estatísticas (ENCE) del Instituto Brasileiro de Geografia e Estatística (IBGE), anteriormente empleadas por los investigadores. ${ }^{7}$

En relación con los aspectos éticos se siguieron las recomendaciones de la Resolução n ${ }^{\circ} 196 / 96$ y de la Resolução no 304/00 do Conselho Nacional de Saúde, sobre investigaciones que incluyen seres humanos y en especial, los pueblos indígenas. El proyecto de investigación fue analizado y aprobado por parte del Comité de Ética en Pesquisa de la 
Escola Nacional de Saúde Pública, Fundação Oswaldo Cruz y por la Comissão Nacional de Ética em Pesquisa (Processo n. 596/2004). También fue aprobado por los Consejos Local y Distrital de Salud del Distrito Sanitário Especial Indígena Vilhena.

\section{Resultados}

A continuación se presentan los resultados considerando previamente el número y las características de las mujeres entrevistadas, el nivel y la estructura de la fecundidad, el calendario reproductivo y los determinantes de la fecundidad.

Se entrevistaron 88 mujeres Suruí, que representan $95 \%$ del total de aquellas de 10 años o más, residentes en las aldeas Linha 14 y Placa. En cuanto a la representatividad según distribución por edad, de las 88 mujeres, $48,8 \%$ tenían entre $10-19$ años, $22,7 \%$ entre $20-29$ años, $17,1 \%$ entre $30-39$ años, $2,3 \%$ entre $40-49$ años y $9,1 \%$ de 50 y más años.

Se observa que el nivel de escolaridad de las mujeres Suruí es bajo, concentrándose entre 1 y 4 años de estudio; $19,7 \%$ nunca fueron a la escuela, $46,1 \%$ tenían hasta 4 años de estudio, 19,7\% habían completado cinco años, $13,1 \%$ habían hecho 6 a 8 años y $1,3 \%$ enseñanza media.

La tasa bruta de natalidad (TBN) en 2003 fue 40,6 por 1000 habitantes y en 2004 fue 59,04 por 1000 habitantes, para un promedio móvil 2003-2004 de 49,8 por 1000 habitantes. La tasa de fecundidad general fue 250 por 1000 mujeres en edad fértil (MEF, 15-49 años) en 2003-2004. La tasa de fecundidad total fue 6,3 hijos. La moda en cuanto al número deseado es de 4 hijos por mujer y la media es inferior a 5 hijos por mujer $(4,7)$.

La curva de las tasas específicas de fecundidad muestra la actividad reproductiva de las mujeres en cada grupo de edad, con un patrón compuesto por los grupos de edad de inicio, de cúspide y de declinación (Figura 1). El comienzo de la procreación se sitúa en el grupo de 15 a 19 años y una gran cúspide en el grupo etáreo de 20 a 24 años. En el año 2004, las mujeres no tuvieron nacidos vivos después de los 35 años: por eso, la declinación de la fecundidad específica en el 2004 fue a los 34 años (en el 2003 había declinado a los 40 años). La fecundidad acumulada fue de 6,5 hijos por mujer y expresa la experiencia, es decir, la historia de la fecundidad a lo largo de los años reproductivos.

\section{Figura 1}

Tasas específicas de fecundidad en mujeres Suruí, Rondônia, Brasil, 2004.

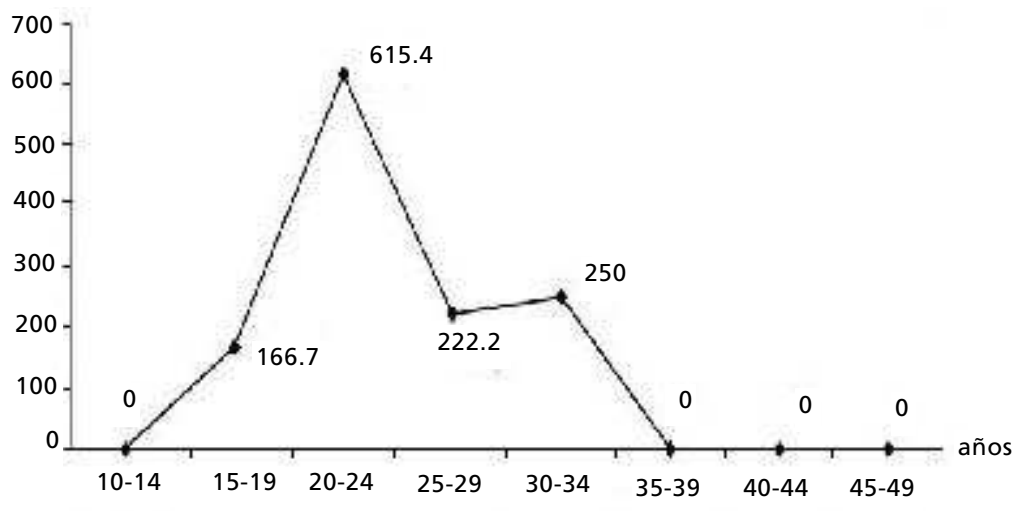

\section{Calendario reproductivo - timing}

La edad promedio de la menarquía expresada por las mujeres fue 12 años y la edad calculada fue 11,8 años, con edades mínima de 9 y máxima de 15 años. La proximidad de las edades expresada y calculada habla del buen recuerdo de las mujeres acerca de los eventos reproductivos.
El matrimonio es prácticamente universal en la sociedad Suruí. Los resultados indican que, en el grupo etario de 20 a 24 años, una única mujer de 20 años se encontraba sola sin marido, todas las demás estaban casadas. La poligamia es una práctica común en la sociedad Suruí: entre las mujeres unidas, más de la mitad $(55,2 \%)$ vivían en uniones poligámicas (es decir, estaban casadas con hombres que tenían 
dos o más mujeres).

La edad promedio de la entrada a la unión fue 13,8 años, similar a lo encontrado en los Xavante entre 1993 y 1997 (Tabla 1), con edades mínima de 10 y máxima de 20 años (Figura 2). Casi 70\%
$(69,6 \%)$ de las mujeres entran a la unión entre 12 y 14 años y $83,9 \%$ antes de 16 años; lo anterior quiere decir que la entrada a la unión es masiva y mayoritaria hasta los 15 años. Todas entraron hasta los 20 años (valor máximo)

Tabla 1

Comparaciones en el calendario reproductivo (timing) de mujeres Suruí, Rondônia, Brasil, 2004 y 2005, en comparación con los Xavante y los Yanomami.

\begin{tabular}{lccc}
\hline & Suruí 2004 & Xavante 1993-1997* & Yanomami 1958-1986 ** \\
\hline Promedio de edad de entrada a la unión (años) & 13.8 & $13-14$ & 12.4 \\
Promedio de edad de inicio de la primera & 15.2 & \\
gestación (años) & & 23.3 (interpartal) \\
Promedio de edad al nacimiento del primer hijo & 15.7 & $24-41$ \\
Promedio de intervalos (meses) & 22.8 & \\
& (p.ingravidez) & & \\
Promedio de meses de lactancia & 17.9 & &
\end{tabular}

*Souza y Santos, 20019; **Early y Peters, 1992.3

\section{Figura 2}

Edad de entrada a la unión de las mujeres Suruí, Rondônia, Brasil, 2004.

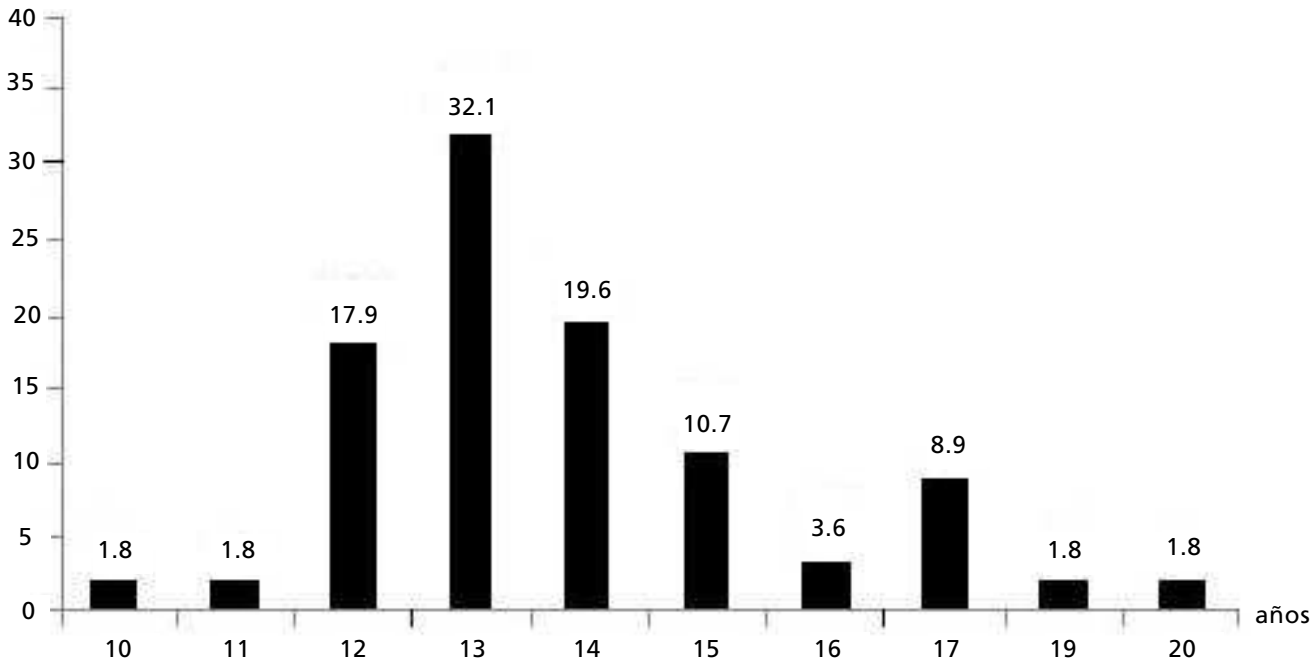

El intervalo promedio entre la menarquía y la entrada a la unión fue 17,3 meses, es decir, las jóvenes Suruí se casan aproximadamente un año y medio después de la primera menstruación. No obstante, casi la cuarta parte $(24,4 \%)$ entra a la unión inmediatamente después de la menarquía. Todas las mujeres unidas lo hicieron en un máximo de cinco años después de la menarquía.

Las edades de inicio de la primera gravidez y del nacimiento del primer hijo (primer parto) son tempranas (Figuras 3 y 4). Ambos promedios de edad se sitúan alrededor de los 15 años. La edad de la madre al inicio de la primera gravidez se sitúa 
básicamente entre los 12 y los 17 años, con promedio de 15,2 años y edad máxima de 22 años. Debe resaltarse que en 9,6\% de las mujeres fue a los 20 años y más (Figura 4). Respecto de la edad al primer hijo (primer parto) el promedio fue 15,7 años, un año menos que las Xavante $(16,8)$ (Tabla 1) $90,7 \%$ de las mujeres Suruí lo tuvieron antes de 20 años de edad; de acuerdo con lo anterior, casi todas serían "madres adolescentes", si aplicáramos dicho criterio occidental. Si se consideran multíparas las mujeres que han tenido 3 hijos o más, $40 \%$ de ellas lo eran, $20 \%$ habían tenido más de 6 partos; todas las mujeres con 50 años y más tuvieron más de 6 partos, con un valor máximo de 17.

Entre los Suruí, el parto tenía lugar tradicionalmente en una pequeña cubierta de paja de palma especialmente construida para este fin. Esto porque, como en el caso de la menstruación, el parto está también rodeado de prescripciones y prohibiciones que, entre otros aspectos, pretende impedir el contacto entre hombres y demás niños del domicilio con sangre asociada con el parto. En esta pequeña casa la mujer cumple con el período de resguardo (postparto), que se puede prolongar por más de un mes, durante el cual está rodeada de cuidados especiales provistos generalmente por las mujeres más viejas de la casa. ${ }^{14}$ Con la creciente exposición de los Suruí a la medicina occidental, se han producido cambios en los comportamientos asociados con el parto y el postparto. Los resultados indican que, entre los 181 partos reportados por las mujeres entre 20-40 años, 50,3\% tuvieron lugar en la aldea y $49,7 \%$ en hospitales. Ya para las mujeres mayores de 40 años, los porcentajes fueron de $82,5 \%$ en la aldea

\section{Figura 3}

Edad al inicio de la primera gravidez de las mujeres Suruí, Rondônia, Brasil, 2004

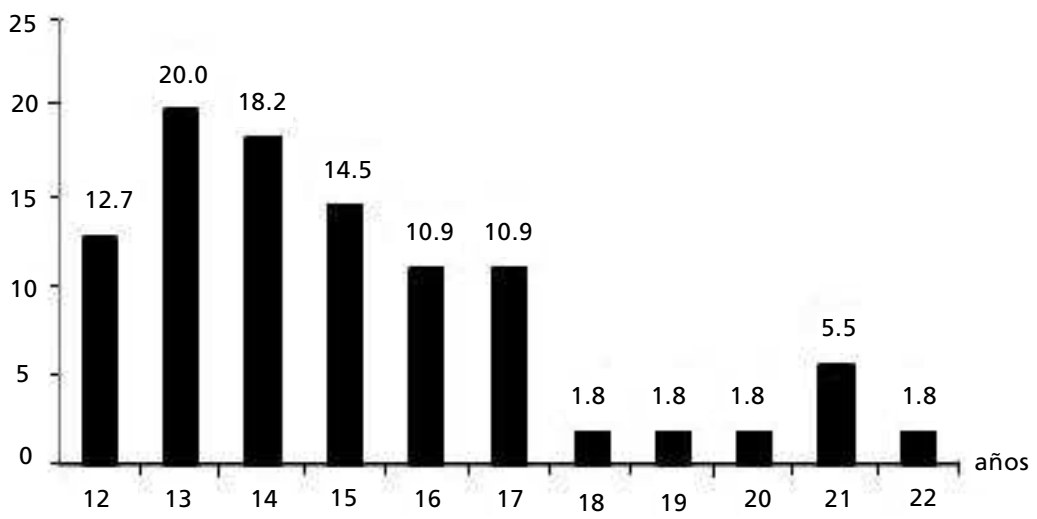

Figura 4

Edad de nacimiento del primer hijo de las mujeres Suruí, Rondônia, Brasil, 2004.

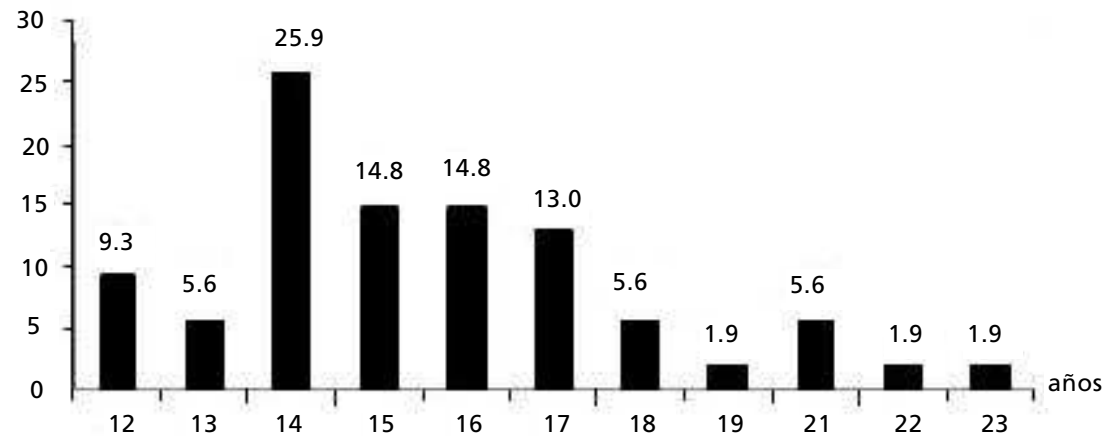


y $17,5 \%$ en hospitales. Esto indica un cambio en las últimas décadas.

Se analizó la relación entre los años de escolaridad y la edad de las madres de 15 a 35 años al nacimiento del primer hijo y no parecen tener relación (Tabla 2). Por lo tanto, el comportamiento reproductivo persiste en forma fuerte aún cuando las mujeres logren avances en el acceso a la escolaridad formal.

Tabla 2

Edad al primer hijo según escolaridad de las madres Suruí de 15 a 35 años, Rondônia, Brasil, 2004 y 2005.

\begin{tabular}{|c|c|c|c|c|c|}
\hline \multirow{3}{*}{$\begin{array}{l}\text { Escolaridad } \\
\text { (años) }\end{array}$} & \multicolumn{4}{|c|}{ Edad al primer parto (años) } & \multirow[t]{3}{*}{ Total } \\
\hline & \multicolumn{2}{|c|}{$12-15$} & \multicolumn{2}{|c|}{$16-19$} & \\
\hline & $\mathrm{N}$ & $\%$ & $\mathrm{~N}$ & $\%$ & \\
\hline $0-3$ & 14 & 66,7 & 7 & 33,3 & 21 \\
\hline $4-8$ & 8 & 61,5 & 5 & 38,5 & 13 \\
\hline Total & 22 & & 12 & & 34 \\
\hline
\end{tabular}

La proporción de mujeres mayores de 10 años que estaban solteras fue $35,2 \% ; 64,5 \%$ tenían entre 10 y 14 años de edad. La edad media y mediana para estar soltera fue de 14 años, con edad mínima de 10 años y máxima de 21 años.

El promedio de amamantación fue de un año y medio (18,4 meses). La mayoría, 60,4\%, refirió haber amamantado entre 12 a 23 meses, $22,9 \%$ amamantó durante 2 a 3 años y sólo $14,6 \%$ refirió haber dado el pecho al niño durante 6 a 11 meses. La moda fue de 24 meses, lo cual significa que la norma es dar el pecho al niño durante los dos primeros años de vida. El promedio mínimo por mujer fue 8 meses (o sea, una dada mujer amamantó sus hijos, en media, por lo menos 8 meses), lo cual es digno de resaltar porque, además, ninguna madre dio pecho al niño por menos de 6 meses. Aunque sea difícil captar la información, las entrevistas sugieren que la amamantación permanece en forma exclusiva, por lo menos hasta los 6 meses de vida del niño, aunque en tiempo atrás era más prolongada.

La importante presencia de la alimentación materna en la cultura Suruí, unida con la abstinencia sexual postparto que las mujeres continúan practi- cando, contribuye en el espaciamiento de las gestaciones. Este hallazgo es consistente con los intervalos intergenésicos como veremos adelante.

Respecto de las mujeres Suruí, durante las entrevistas se realizaron muchas indagaciones sobre el uso de métodos anticonceptivos. A pesar de lo anterior, se encontró que su uso es poco - solamente una de las mujeres usaba anovulatorios orales. Hubo también el relato de dos mujeres $(4,8 \%)$ a quienes se les había practicado histerectomía, una de ellas por miomas; y también a dos mujeres $(4,8 \%)$ les habían practicado ligadura de trompas. Dos mujeres $(4,8 \%)$ refirieron haber utilizado "remédio do mato" ("remedio del monte") para no tener más hijos. A partir de las entrevistas queda evidente que, aunque el deseo por el uso de contraceptivos sea referido por muchas mujeres, persiste la valorización por familias grandes, con la presencia de muchos hijos. El deseo de más hijos tiene variaciones así: la mayoría, $67,3 \%$, desea tener más, $20,7 \%$ de ellas no desean más hijos y $12 \%$ no lo saben.

Otro importante determinante de la fecundidad es el intervalo intergenésico (IIG). Se toma aquí como período de ingravidez y su promedio resume el comportamiento reproductivo. El promedio general se situó cerca de los dos años (22,8 meses), valor cercano a los Xavante (23,3 meses) y menor que en los Yanomami (24-41 meses) (Tabla 1); la mediana fue 21 meses y la moda 18 meses (Figura 5). Si consideramos que los IIG están relacionados directamente con la mortalidad infantil y en la infancia, tanto en forma individual como colectiva, el hecho de que el promedio sea superior a 18 meses es altamente significativo, tanto para la sobrevivencia y la calidad de los cuidados que se prodigan a los niños, como para favorecer la recuperación de la madre, entre una y otra gestación. Casi $60 \%$ $(59,2 \%)$ de las mujeres que tenían más de una gestación tuvieron promedios de IIG entre 12 y 23 meses, lo cual, aplicado al ámbito colectivo, puede favorecer una disminución en la mortalidad. 


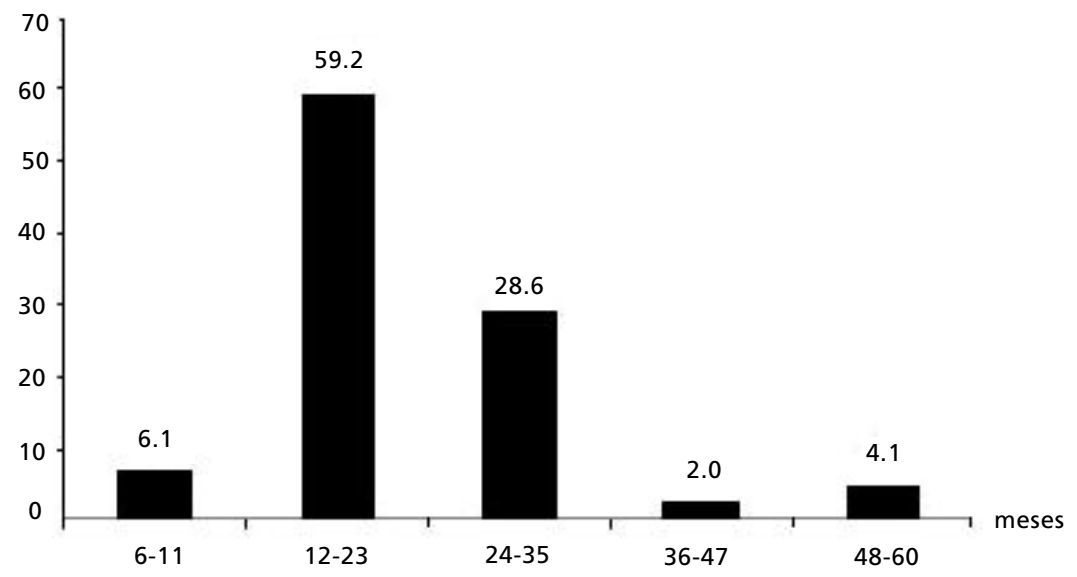

Mujeres con dos o + gestaciones.

\section{Discusión}

En los últimos años ha habido una expansión de los estudios sobre la demografía y la salud reproductiva de los pueblos indígenas en Brasil.6,8 Aunque se necesita mucha cautela en la realización de generalizaciones, pues es grande la diversidad sociocultural indígena, hay algunos aspectos consistentes. Uno de ellos es que los niveles de fecundidad tienden a ser elevados, resultando en poblaciones compuestas por hasta la mitad de niños y adolescentes. En el caso Suruí, con una TFT próxima a 6 hijos, los resultados señalan una fecundidad menos elevada en comparación con otros pueblos indígenas de Brasil, ya que hay diversos estudios que reportan TFT superiores a 7 hijos por mujer. ${ }^{6}$

En la estructura de la fecundidad es esencial considerar explícitamente los llamados determinantes próximos, o variables intermedias, denominadas factores intrínsecos, es decir, aquellas que influyen sobre el nivel de fecundidad. ${ }^{20}$ Estas se refieren a los niveles, a los diferenciales y a los determinantes que inciden de manera directa e indirecta en la reducción o en el aumento de la fecundidad y que actúan de manera intermedia entre la organización social y los demás factores sociales, culturales, históricos y económicos. ${ }^{21}$ El modelo más conocido identifica los siguientes determinantes próximos: a) proporción de mujeres casadas (patrón marital); b) uso de anticonceptivos; c) aborto inducido y d) amamantación, que, en conjunto con el tiempo de postparto, constituye un período de infecundabilidad.22 Las llamadas variables de calendario (timing), indicadoras del comportamiento reproductivo, se volvieron fundamentales en los estudios demográficos que abordan la toma de decisiones reproductivas.

A partir de los resultados de la indagación a las mujeres Suruí se buscó realizar un análisis exploratorio de algunos de los determinantes antes indicados. Reconocemos que los datos necesitan analizarse con cautela, pues se basan en entrevistas $y$, en el caso de las mujeres indígenas, la cuestión de la cuantificación de los eventos demográficos presenta particular complejidad, no solamente asociada con el factor de memoria, sino también con la cuestión de cuantificar eventos vitales que pueden haber ocurrido décadas atrás. 6 Hechas estas salvedades, el patrón de alta fecundidad de las mujeres Suruí se relaciona con un inicio de la vida reproductiva inmediatamente después de la menarquía, con intervalos intergenésicos cortos y baja utilización de contraceptivos. Por otro lado, persiste en la cultura Suruí una fuerte valorización de la amamantación y la manutención de la abstinencia sexual postparto entre las mujeres.

La regla general, como ya se refirió, es no utilizar ningún método anticonceptivo. Sin embargo, hay una demanda que parece estar desatendida. Es el caso de una madre con muchos hijos y que refirió muchos casos de enfermedades en su familia, especialmente tuberculosis y que, viene usando anticon- 
ceptivos orales hace algunos años sin prescripción médica y sin seguimiento por parte de programa alguno. Cuando la enfermera revisó la forma como aquélla toma el medicamento, observó que no seguía los procedimientos recomendados para su uso. Debe resaltarse que ella hacía el esfuerzo económico y tenía manchas típicas de anticonceptivos orales en el rostro. Otro caso observado fue el de un hombre que pidió a su mamá que le diera "remedio del monte" para su mujer, cuando tenía 7 hijos, pero, ella no le quiso dar, según él se quejó.

Aunque las características demográficas de las mujeres Suruí presentan importantes paralelos con las que vienen siendo descritas en la literatura para otros pueblos indígenas, los resultados de este estudio señalan aspectos particulares que merecen atención. Dicen respecto, por ejemplo, de los crecientes niveles de escolaridad formal de las mujeres Suruí, al uso de contraceptivos y a la medicalización del parto. Estos aspectos han sido poco investigados en el contexto de pesquisas demográficas sobre los pueblos indígenas en Brasil $y$, ciertamente, merecen recibir mayor atención. En relación con el parto, por ejemplo, Coimbra Jr., ${ }^{14}$ con base en datos recolectados en los años 1980, no reportó partos que fueran atendidos en hospitales. Se trata de un patrón bastante distinto del observado en este estudio, dos décadas después.

Considerando la centralidad de la dimensión reproductiva en la vida de las mujeres Suruí, se refuerza la importancia de cualificar los servicios de atención a la salud de la mujer. Oliveira et al.23 condujeron una evaluación de la atención en el período prenatal de las mujeres Suruí a partir de los registros disponibles en la "Casa de Saúde do Índio" en el período de 2000-2004. Dichos autores evidenciaron una baja priorización por parte de los servicios de salud disponibles para los Suruí en la asistencia prenatal. En cuanto al perfil de las mujeres, la semejanza de lo observado durante las entrevistas realizadas en las dos aldeas, la edad materna media verificada en los prontuarios fue de 25 años y $42 \%$ de ellas eran menores de 20 años. Se observaron bajas frecuencias de consulta por mujer durante la gestación - apenas 1,3 consultas en promedio realizadas, aunque el programa nacional prevé al mínimo seis consultas de prenatal, siendo al menos una realizada en el primer trimestre de embarazo. La mayoría de las consultas se realizaran solamente al quinto mes. En relación con los exámenes de laboratorio considerados como básicos por el Programa de Humanización en el Prenatal y Nacimiento, 24 no hubo registro en $76,4 \%$ de los prontuarios analizados (no se realizaron serología para virus de la inmuno- deficiencia humana (VIH) ni hepatitis B, de acuerdo con el protocolo del programa). Además, $60,8 \%$ de las gestantes no realizaron todos los procedimientos clínico-obstétricos reglamentarios. De igual importancia es la constatación de completa falta de atención a la mujer y al recién nacido en la primera semana después del parto, según lo previsto por el programa nacional. Por lo tanto, se constató que las prescripciones y normas establecidas por el Ministerio de Salud brasilero para la asistencia prenatal 24 no se realizan en forma satisfactoria en el caso de las mujeres Suruí. Esa conclusión pone en evidencia la situación de desigualdad con respecto al acceso a los servicios de salud por parte de los indígenas en comparación a la población brasilera en general.

Coimbra e Garnelo 8 observan que, en la mayoría de las veces, los servicios de salud destinados a la atención de los pueblos indígenas no se encuentran preparados, tanto en lo que se refiere a la disponibilidad de recursos humanos como de infraestructura física, para proveer atención especializada en las áreas da salud reproductiva y salud de la mujer. Complementan los autores: 8

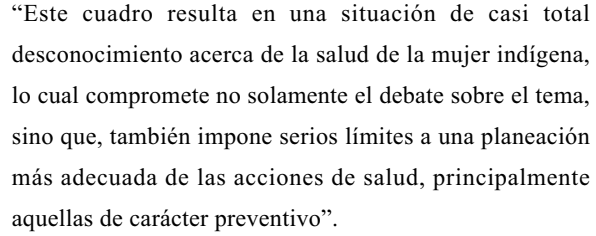

Conforme lo que señalan Pagliaro et al.,6 una de las principales dificultades para la realización de estudios demográficos sobre los pueblos indígenas se refiere a las fuentes de datos. El presente estudio sobre los Suruí se apoyó en recolección de datos primarios en las aldeas. A pesar de estas dificultades, es evidente que el cuadro que emerge es bastante informativo, inclusive provee informaciones útiles para la planeación de las acciones de salud.

\section{Agradecimientos}

El equipo de investigación agradece a todos los funcionarios del Polo Base Cacoal, especialmente a Mariana Kely Lima y a Maria Marcilene dos Santos, así como a los estudiantes de la Universidad de Rondônia, especialmente a Rosana Feitosa. De igual manera agradece el apoyo del Centro de Estudos em Saúde do Índio en Rondônia (CESIR) de la Universidad Federal de Rondônia y el apoyo financiero de la Fundación Ford. 


\section{Referencias}

1. Howell N. Demographic anthropology. Annu Rev Anthropol. 1986; 15: 219-46.

2. Kertzer D, Fricke T (editores). Anthropological demography. Toward a new synthesis. Chicago: University of Chicago Press; 1997.

3. Early J, Peters J. The population dynamics of the Mucajai Yanomami. San Diego: Academic Press; 1992.

4. Coimbra Jr. CEA, Flowers NM, Santos RV, Salzano FM. The Xavánte in transition: health, ecology, and bioanthropology in Central Brazil. Ann Arbor: University of Michigan Press; 2002.

5. IBGE (Instituto Brasileiro de Geografia e Estatística). Tendências demográficas. uma análise dos indígenas com base nos resultados da amostra dos censos demográficos 1991 e 2000. Rio de Janeiro, RJ; 2005.

6. Pagliaro H, Azevedo MM, Santos RV (organizadores) Demografia dos povos indígenas no Brasil. Rio de Janeiro: Editora Fiocruz; 2005.

7. Arias MM. Determinantes próximos de la fecundidad Comportamiento reproductivo de las indígenas Chamibida de Antioquia, Colombia. Cad Saúde Pública. 2005; 21: 1087-98.

8. Coimbra Jr. CEA, Garnelo L. Questões de saúde reprodutiva da mulher indígena no Brasil. In: Monteiro S, Sansone L, organizadores. Etnicidade na América Latina: um debate sobre raça, saúde e direitos reprodutivos. Rio de Janeiro: Editora Fiocruz; 2004. p. 153-74.

9. Souza LG, Santos RV. Perfil demográfico da população indígena Xavánte de Sangradouro-Volta Grande, Mato Grosso, Brasil (1993-1997). Cad Saúde Pública. 2001; 17: 355-65.

10. Coimbra Jr. CEA, Santos RV. Saúde, minorias e desigualdade: algumas teias de inter-relações, com ênfase nos povos indígenas. Cienc Saúde Coletiva. 2000; 5: 125-32.

11. Santos RV, Coimbra Jr. CEA. Cenários e tendências da saúde e da epidemiologia dos povos indígenas no Brasil. In: Epidemiologia e saúde dos povos indígenas no Brasil. Coimbra Jr CEA, Santos RV, Escobar AL (orgs). Rio de Janeiro: Editora Fiocruz; 2003. p. 13-47.

12. Santos RV, Coimbra Jr CEA. On the (un)natural history of the Tupí-Mondé Indians. Bioanthropology and change in the Brazilian Amazonia. In. Toward a biocultural synthesis: political-economic perspectives in biological anthropology. Goodman A, Letherman T (editores). Ann Arbor: Michigan University Press; 1998. p. 269-94.
13. Mindlin B. Nós Paiter: Os Suruí de Rondônia. Petrópolis: Vozes; 1985

14. Coimbra Jr. CEA. From shifting cultivation to coffee farming: the impact of change on the health and ecology of Suruí Indians in the Brazilian Amazon [thesis]. Bloomington: Indiana University; 1989.

15. Orellana JDY, Coimbra Jr. CEA, Lourenço ALP, Santos RV. Estado nutricional e anemia em crianças Suruí, Amazônia, Brasil. J Pediatr (Rio J). 2006; 82: 383-8.

16. Lourenço ALP, Santos RV, Orellana JDY, Coimbra Jr. CEA Nutrition transition in Amazonia: Obesity and socioeconomic change in the Suruí Indians from Brazil. Am J Hum Biol. 2008; 20:564-71

17. Basta PC, Coimbra Jr CEA, Escobar AL, Santos RV, Alves LCC, Fonseca LS. Survey for tuberculosis in an indigenous population of Amazonia: the Suruí of Rondônia, Brazil. Trans R Soc Trop Med Hyg. 2006; 100: 579-85.

18. Miranda-Ribeiro A, Rios-Neto ELG, Carvalho JAM Reconstrução de histórias de nascimentos a partir de dados censitários: uma análise comparativa de duas metodologias. Rev. Bras. Estud Popul. 2009; 26: 21-36.

19. Arias MM. Reproducción y cultura: pervivencia y perspectiva de futuro de las etnias de Antioquia - Colombia [tese]. Rio de Janeiro: Escola Nacional de Saúde Pública, Fundação Oswaldo Cruz; 2002.

20. Preston S. The changing relation between mortality and level economic development. Popul Stud (Camb). 1975; 29: $157-248$.

21. Rico VJ. Demografía social y salud pública. Cali: Universidad del Valle; 1993

22. Bongaarts J. A framework for analysing the proximate determinants of fertility. Popul Dev Rev. 1978; 41: 105-32.

23. Oliveira MVG, Escobar AL, Coimbra Jr CEA. Avaliação do pré-natal em indígenas Suruí, Rondônia. Trabalho apresentado no $57^{\circ}$. Congresso Brasileiro de Enfermagem, Goiânia, Goiás. Disponível em: http://bvsms.saude.gov.br/bvs/publicacoes $/ 57 \mathrm{cbe} /$ resumos $/ 1030 . \mathrm{htm}$

24. Programa de Humanização no Pré-Natal e Nascimento. Disponível em: http://portal.saude.gov.br/portal/arquivos/ pdf/ manual_puerperio_2006.pdf

Recebido em 14 de abril de 2009

Versão final apresentada em 23 de abril de 2010

Aprovado em 7 de junho de 2010 